Die Wahl von Ignazio Cassis zum Präsidenten des im April dieses Jahres gegründeten neuen Krankenkassenverbands Curafutura hat auch in der Ärzteschaft für Gesprächsstoff gesorgt. Im folgenden Artikel erläutert der Nationalrat, ehemalige Tessiner Kantonsarzt und ehemalige Vizepräsident der FMH, warum er sich zu diesem Schritt entschlossen hat und warum es ihm wichtig ist, sich als Arzt in diesem Bereich zu engagieren. Seine Ausführungen scheinen uns ein begrüssenswerter Beitrag zu einer transparenten Kommunikation und offenen Diskussion, und in diesem Sinn danken wir Ignazio Cassis für die Darlegung seines Standpunkts.

Die Redaktion

\title{
curafutura blickt in die Zukunft
}

Ignazio Cassis

Präsident curafutura
Curare heisst behandeln, betreuen, pflegen; futuro bedeutet Zukunft. Curafutura steht somit für eine zukunftsorientierte Versorgung und ist Name wie Programm eines neugegründeten Verbands, dessen Präsident ich bin. Curafutura ist Ende April von den drei grossen Krankenversicherern CSS, Helsana und Sanitas gegründet worden und hat kürzlich mit der KPT bereits ersten Zuwachs bekommen. Diese vier Versicherer werden ab 2014 nicht mehr Mitglied des Krankenversicherungsverbandes santésuisse sein. Welche Konsequenzen hat diese Entwicklung für das Gesundheitswesen in der Schweiz?

Ich bin Facharzt für Innere Medizin sowie für Prävention und Gesundheitswesen und habe jahrelang im Tessin als Kantonsarzt gearbeitet. Ich war Präsident der Schweizerischen Gesellschaft für Public Health und Vizepräsident der FMH. Zudem engagiere ich mich für Prävention und Gesundheitsförderung bei der Gesundheitsstiftung Radix sowie für eine qualitativ hochstehende Betreuung der Bewohnerinnen und Bewohner von Pflegeheimen beim Verband CURAVIVA Schweiz. Gemäss Terminologie des Krankenversicherungsgesetzes komme ich also aus der Welt der Leistungserbringer.

\section{«Dass ich nun beide Seiten - und auch noch den Staat - vertrete, kommt nicht überall gut an.»}

Korrespondenz:

Dr. med. Ignazio Cassis, MPH Via dei Lucchini 14 CH-6926 Montagnola ignazio.cassis[at]parl.ch

\section{Fronten aufweichen statt zementieren}

Ich bin auch Politiker und engagiere mich als FDPNationalrat seit Jahren für eine nachhaltige Gesundheitspolitik in unserem Land. Und jetzt auch noch Präsident von curafutura. Das wirft Fragen auf und reaktiviert das - absurde - Feindbild zwischen Versicherern und Leistungserbringern. Meine Motivation ist: Ich will etwas bewegen. Schliesslich habe ich schon im Militärdienst gelernt: «Wer ein Problem identifiziert und nichts zu dessen Lösung beiträgt, wird Teil des Problems.»
Als Bürger und Versicherter hat mich das Verhalten der Krankenversicherer oft irritiert. Als Standespolitiker bei der FMH habe ich die Versicherer kritisiert, weil Lösungen für dringende Probleme auf die lange Bank geschoben wurden. Und jetzt? Auf einmal habe ich die Möglichkeit, in der Branche etwas zu bewegen. Was nun also? Weiterhin kritisieren oder zupacken? Ich habe mich für Letzteres entschieden. Das ist zwar risikoreich, aber so sieht Verantwortung übernehmen aus.

Dieser Entscheid hat mir gerade in meinem Heimatkanton Tessin nicht nur Freunde eingebracht. Eine «Entweder-oder-Einstellung», kombiniert mit Vorurteilen, gipfelte rasch einmal in der Forderung «Entweder das Tessin oder die Krankenkassen». Auch in der Romandie waren einige Reaktionen ziemlich heftig, ganz nach dem Motto «Man sollte nicht mit seinem Feind tanzen». In der Deutschschweiz gab es zwar Skepsis, aber auch vorsichtigen Optimismus. «Schauen wir einmal, was der bringt», war dort die Devise.

Die Ursache dieser teilweise heftigen Reaktionen ist leicht zu finden. Der Zeitgeist will es, dass die Krankenversicherer per Definition die «Bösen» sind, während die Leistungserbringer in jedem Fall zur Fraktion der «Guten» gehören. Dass ich nun beide Seiten - und auch noch den Staat - vertrete, kommt nicht überall gut an. Schliesslich soll man in einem Krieg doch bitteschön klar sagen, für welche Armee man kämpft.

\section{Damit die Verstaatlichung nicht von selbst kommt}

Nur, im Falle unseres Krankenversicherungsgesetzes (KVG) ist die kriegerische Metapher fehl am Platz. Mit der Einführung des KVG hat sich das Schweizer Volk nämlich für Verhandlungspartner und nicht Verhandlungsgegner entschieden. Mit einem klaren Auftrag an die involvierten Parteien, die anstehenden Probleme zu guten Lösungen zu führen, und zwar mit Verträgen, welche die Details der Zusammenarbeit und nicht diejenigen des Krieges regeln. Die Idee: Der Staat sorgt für die - liberalen - Rahmen- 


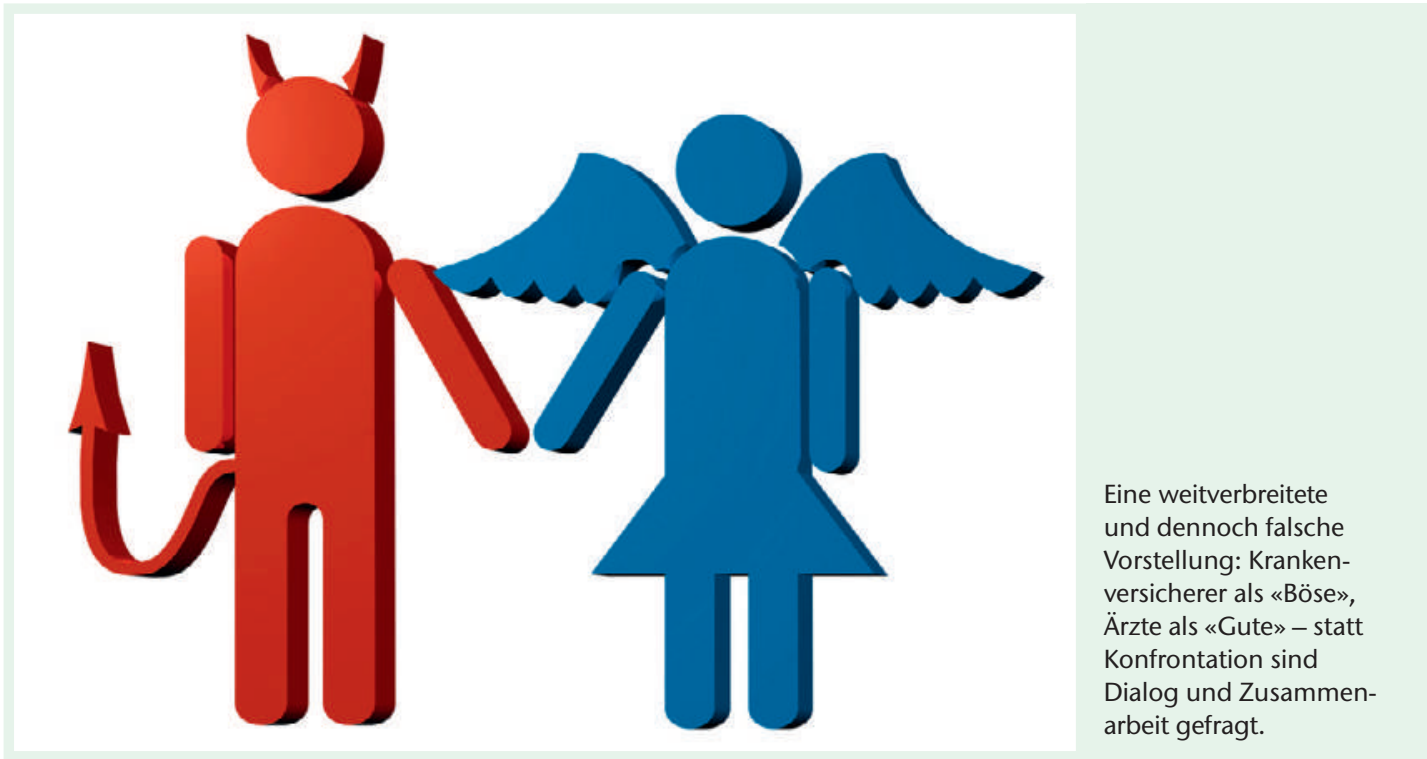

bedingungen und nimmt die Aufsicht über das System wahr, die Leistungserbringer und Krankenversicherer arbeiten als echte Partner zusammen - zum Wohle der Prämienzahler und Patienten. Sind sie dazu nicht in der Lage und lähmen Blockaden die Weiterentwicklung des Systems, so ist der Staat zum Eingreifen gezwungen. Und solche Blockaden gab es in der Vergangenheit zu viele. Die Folge davon ist vor allem für die Patienten negativ: Das System wird ganz oder teilweise verstaatlicht, und zwar unabhängig davon, was Bürgerinnen und Bürger bei der anstehenden Volksabstimmung über die Einheitskasse beschliessen werden. Die Verstaatlichung kommt sozusagen von selbst. sundheitssystem zu den besten der Welt machen, nicht sinnlos gefährden. Dazu gehört, dass wir unsere Verantwortung gegenüber den Versicherten und Patienten wahrnehmen - mit innovativen Versorgungsmodellen und nicht mit der Selektion guter Risiken. Dazu gehört auch, dass wir uns gegen ein von der Politik bis ins Detail reguliertes und von Beamten dominiertes System wehren.

Als Präsident der curafutura nehme ich diese Herausforderung gerne an. Was mich dazu treibt? Die Überzeugung, dass wir es selber in der Hand haben, unser Gesundheitssystem schrittweise zu reformieren, und wir gegenüber Patienten und Steuerzahlern die Pflicht haben, diese zentrale Frage anzupacken.

\section{«Ich sehe meine Aufgabe als Präsident von curafutura darin, den Dialog zwischen allen am Krankenversicherungssystem beteiligten Partnern wieder in Gang zu bringen.»}

\section{Ein gutes System nicht sinnlos gefährden}

Entsprechend sehe ich meine Aufgabe als Präsident von curafutura darin, den Dialog zwischen allen am Krankenversicherungssystem beteiligten Partnern wieder in Gang zu bringen. Ich will erreichen, dass wir die gleiche Sprache sprechen, Vorurteile hinter uns lassen und dafür sorgen, dass wir die Errungenschaften, die unser liberales, wettbewerbliches Ge-
Dabei ist aber eines sicher: Alleine werde ich diese Aufgabe nicht meistern können. Ich brauche die Unterstützung der Verhandlungspartner, der Versicherer und der Leistungserbringer. Und ich brauche die Unterstützung all jener Versicherten, die auch in $\mathrm{Zu}-$ kunft ein leistungsfähiges Gesundheitssystem wollen, das sie medizinisch bestens versorgt. 